\title{
A Hybrid Approach for CBIR using SVM Classifier, Partical Swarm Optimizer with Mahalanobis Formula
}

\author{
Amit Singh \\ M.Tech Scholar, \\ Department of CSE \\ LNCTS Bhopal, (INDIA)
}

\author{
Parag Sohoni \\ Department of Computer Sc. and \\ Engineering \\ LNCTS Bhopal, (INDIA)
}

\author{
Manoj Kumar \\ Department of Computer Sc. and \\ Engineering, \\ LNCTS Bhopal (INDIA)
}

\begin{abstract}
The goal of content-based image retrieval is to retrieve the images that as per the user query. Mainly the Content based Image retrieval technique attempt to search through the database that finds images that are perceptually similar to a given query image. Set of low-Level visual features (Color, Shape and Texture) are used to represent an image in most modern content based image retrieval systems. Therefore, a gap exists between low-level visual features and information of high-level perception, which is the main reason that down the improvement of the image retrieval accuracy. To retrieve several features of images and shorten the semantic gap between low-level visual feature and high-level perception a Hybrid support vector machine (SVM) scheme is proposed in this paper. Image data set is taken from coral image data set.
\end{abstract}

\section{Keywords}

CBIR, SVM, PSO, Semantic gap, CCM, distance measurement.

\section{INTRODUCTION}

Image retrieval is a way for retrieving, searching and browsing images from a big database of digital images. Most of the conventional and common methods of image retrieval use some methods of adding metadata such as keywords, captioning, or explanations to the images to perform the image retrieval over the explanation words. Handbook image annotation technique is the time-consuming, laborious and costly to address. And there has been already a large amount of researches done on involuntary image explanation. Moreover, the enhancements in the semantic web and social web applications have inspired the growth of numerous webbased image annotation tools. Mainly the Content-based image retrieval (CBIR) has attracted much research interest in recent years [1]. In particular, there has been growing interest in indexing biomedical images by content [2]. Handbook indexing of images for content-based retrieval is bulky, error prone, and prohibitively costly [3]. Due to the lack of efficient automated methods, nevertheless, biomedical images are classically annotated manually and retrieved using a text keyword-based search. An ordinary drawback of these systems is that the annotations are indefinite with reference to locations of image features, and text is often inadequate in enabling efficient image retrieval. Even these kind of retrieval techniques are unfeasible for collections of images that have not been explained or indexed. Furthermore, the retrieval of interesting cases, particularly for medical education or building atlases, is a bulky task. Particular CBIR methods that are developed for biomedical images could present the solution to such problems, by this means augmenting the clinical, research, and educational features of biomedicine, designed for any class of biomedical images, on the contrary, it would be essential to grow suitable feature demonstration and similarity algorithms that capture the content in the image. In the figure 1 illustrate the basic functioning process of the CBIR system. Where at first the input data desired as image format then it proceed for the training and extract feature of all images stored in the database. Similarly at parallel get the user query image and do the same feature extraction process and process it for the similarity matching.

The rest part of this paper is organized as follows: Section 2 discussed about the literature review. Section 3 gives detailed description about CBIR. Section 4 described about the proposed methodologies. Section 5 shows the simulation result produced by the proposed algorithm and last section concluded the paper and its future work.

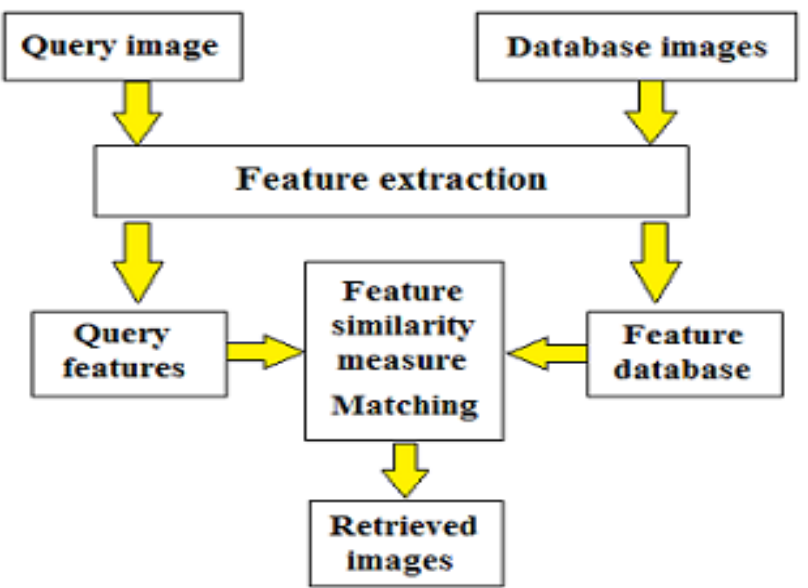

Fig. 1 Block diagram for CBIR System

\section{RELATED WORK}

In earlier, there are so many algorithm has been developed for image retrieval. In this section literature of the related work is described which are developed by different author and researchers:

Monika Daga, Kamlesh Lakhwani et al. proposed a new CBIR classification technique that was developed using the Negative Selection Algorithm of AIS. The MatLab functionalities are being used to extend a fresh CBIR system which has reduced complexity and percentage effectiveness of retrieval is increased depending upon the type of images [15]. Le Hoang Thai, Tran Son Hai, Nguyen Thanh Thuy et al. proposed a method which expresses mutually two areas that are Support Vector Machine (SVM) applying for image classification and Artificial Neural Network (ANN). Firstly, the images are separated into many sub-images based on the extracted features of images. Then each sub-image is 
classified into the responsive class by an ANN. Lastly, SVM has been accumulated all the categorize result of ANN [16]. K. Ashok Kumar \& Y.V. Bhaskar Reddy et al. proposed method, multiple feature distances are combined to acquire image resemblance using classification technology. For managing the noisy positive illustration, a new two step approach is proposed by including the methods of data cleaning and noise tolerant classifier [2]. V. Karpagam1, R. Rangarajan et al. proposed wavelet histograms are used to intend an uncomplicated and well-organized CBIR system with good performance and without making use of any intensive feature extraction technique of image-processing. The wavelet decomposition-based vertical, diagonal and horizontal image attributes and unique indexed color histogram serve as the main features for the retrieval system. Support vector machine is used for classification and thereby to improve retrieval accuracy of the system [3]. A CBIR system based on a multi-scale geometric analysis (MGA) tool, called ripplet transform type-I (RT) have been presented by Chowdhury et al. [4]. Laplacian transform of the sharpened grey-scale image is statistically quantized into colour histogram bins in Malik and Baharudin [5].

\section{CONTENT BASED IMAGE RETRIEVAL}

A typical CBIR system automatically extract visual attributes (color, shape, texture and spatial information) of each image in the database based on its pixel values and stores them in to a different database within the system called feature database $[7,8]$. The feature data for each of the visual attributes of each image is very much smaller in size compared to the image data. The feature database contains an abstraction of the images in the image database where each image is represented by a compact representation of its contents like color, shape, texture and spatial information in the form of a fixed length real-valued multi-component feature vectors or signature. The users usually prepare query image and present to the system. The system automatically extract the visual attributes of the query image in the same mode as it does for each database image and then identifies images in the database whose feature vectors match those of the query image, and sorts the best similar objects according to their similarity value. During operation the system processes less compact feature vectors rather than the large size image data thus giving CBIR its cheap, fast and efficient advantage over text-based retrieval. CBIR classification can be used in one of the two modes. The first mode is exact image matching that is matching two images, one an example image and the other is image in image database. Second mode is approximate image matching which is finding most closely match images to a query image [9].

Basically CBIR used two approaches for retrieving the images from the image data base.

\subsection{Two Approaches}

- Text-based approach (Index images using keywords)

- Content-based approach (Index images using images)

\subsubsection{Text-based Approach:}

Text based method used the keywords descriptions as a input and get the desired output in the form of similar types of images. Examples:- (Google, Lycos, etc.) but Text Based approach [9] have lots of disadvantages over its advantages.

\subsubsection{Content-based Approach:}

Content based approach [9] using image as a input query and it generate the output of similar types of images.

The CBIR is used in various applications such as medical diagnosis, security check, crime prevention etc.

\section{PROPOSED METHODOLOGY}

In this section the discussion about SVM and proposed approaches and their steps followed at the classification time and in the next chapter show the experimental result of the both methods discuss.

\subsection{Overview of SVM}

In machine learning there are two types of methods supervised and unsupervised. Supervised learning based on learn by result and unsupervised based on learn by example. Supervised learning takes input as a set of training data. Support vector machine is a supervised learning technique that analyzes data and identify pattern used for classification. It takes a set of input, read it and for each input desired output form [10] such type of process is known as classification, when if output is continuous than regression performed. For constructing maximum separating hyperplanes SVM maps input vector to a higher dimension feature space. Feature space refers to an input space which is reserved for measuring similarity with the help of kernel function. It is high dimension space where linear separation becomes very easier than input space [14]. In this, raw data is transformed into a fixed length sample vectors. There are two terms which are used in feature space i.e. called feature values and feature vectors. The characteristics of image is called feature values and these feature values presented the machine in a vectors is known as feature vectors. Kernel function used in the kernel method performing some operation such as classification, clustering upon different categories of data like text document, progression, vectors, group of points, image and graphs etc. it maps the input data into a higher dimension feature space because in this data could be easily separated or better structured [14]. There are some points in the feature space which are separated by some distance is called support vectors. It is the point among origin and that point and illustrate the position of the separator. The distance from the decision surface to the closet data point determines the margin the classifier.

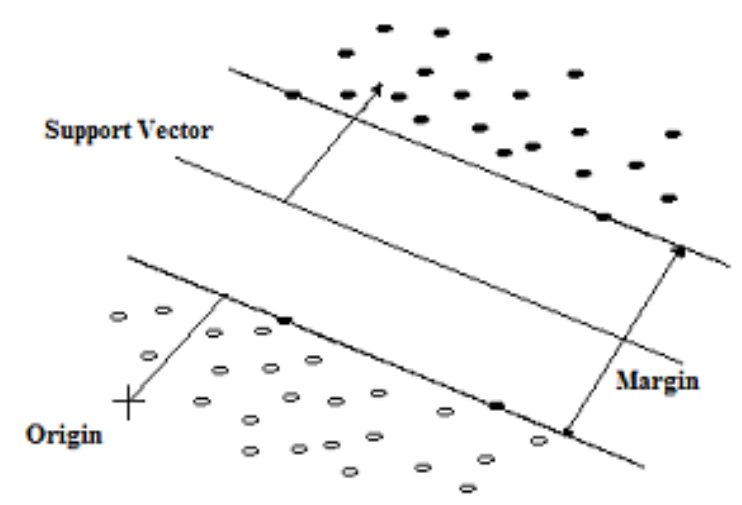

Fig. 2 Linear separating hyper-planes for two class separation

A method of selecting negative samples from the image database has been proposed in [4]. It chose negative samples randomly from the image database. This method is based on the assumption that almost every image in a typical large 
image database is a negative example. But its shortcoming is obvious since not every image is a negative sample [3] proposed a modified approach that used the samples from last query whose similarity scores were less than a threshold as the negative samples for expanding training set. However, the threshold is hard to decide and different queries need different thresholds. Here we propose a new method to append negative samples to the training set automatically and avoid the problem of threshold choosing. The steps can be described as follows:

1) Let $R^{t}$ denote the result list ranked by similarity score after the $t^{t h}$ feedback. There are $\mathrm{M}$ records in $R^{t}$, and each one is represented as $X_{i}$.

$$
R^{t}=\left[x_{1}^{t}, x_{2}^{t} \ldots x_{i}^{t} \ldots x_{M}^{t}\right] .
$$

2) After image query, only top $K$ results are displayed to the user, which is represented as $D_{t}$

$$
D^{t}=\left[x_{1}^{t}, x_{2}^{t} \ldots x_{i}^{t} \ldots x_{K}^{t}\right] \text {. }
$$

In addition, we define $T S^{t}$ as the training set.

$$
T S^{t}=T S^{t} . P \cup T S^{t} . N
$$

Where $T S^{t} . P$ denotes the subset of positive samples in $T S^{t}$, and accordingly, $T S^{t} . N$ is the subset of negative samples in $T S^{t}$. Now the user can select the positive samples from $D^{t}$ and append them into $T S^{t}$.P. The rest of $D^{t}$ are put into $T S^{t} . N$. TS $S^{t} . P$ and $T S^{t} . N$ could be represented as (4) and (5).

$$
\begin{aligned}
& \left.T S^{t} . P=T S^{t}-1 . P \text { \{positive samples in } D^{t}\right\} \\
& \left.T S^{t} . N=T S^{t}-1 . N \text { negative samples in } D^{t}\right\}
\end{aligned}
$$

We define $\mathrm{R}$ as follows:

$$
\mathrm{R}=\frac{\text { the number of positive samples in } T S^{t}}{\text { the number of negative sample in } T S^{t}} .
$$

Our experience indicates that when $r$ is about 0.3 , the SVM classifier obtains good performance. So our system will automatically supply additional negative samples into training set from the tail of $R^{t}$.

$$
\operatorname{numT}=\frac{\operatorname{size}\left(T S^{t} . P\right)}{\mathrm{r}}-\operatorname{size}\left(T S^{t} . N\right)
$$

Where $\operatorname{size}(\mathrm{R})$ is the number of elements in set $\mathrm{R}$. So the negative samples of training set are updated by (8).

$$
\begin{gathered}
T S^{t} . N=T S^{t} . N \cup A N \\
A N=\left\{\text { the last num }{ }^{t} \text { records in } R^{t}\right\} .
\end{gathered}
$$

\subsection{Proposed Algorithm Steps:}

1. Select a source database image containing folder.

2. Extract the color features of each stored images in database.

3. Stored each extracted features into the separate .mat file (DBF_i)

4. Select Query Image

5. Extract feature of query image (QF)

6. Now apply SVM as training purpose.

7. Apply Particle Swarm Optimization technique to optimize the SVM training.
8. Calculate Posterior Probability of each pixel for each DBF_i also for QF.

9. Calculate optimal threshold value of each pixel of each stored images.

10. Compare the values of Posterior Probability with the threshold value.

11. If Posterior Probability $<=$ threshold value

12. Apply Mahalanobis distance formula for similarity matching

13. Classify each of the DBF_i in the respect of QF and use connected components to combine similarity regions

Else

14. Classification on the basis of interior extracted features.

\section{EXPERIMENTAL RESULT}

This section describes the experimental setup of the proposed method and used dataset. For the experimental purpose they used the MATLAB2009A with system configuration of $2.4 \mathrm{GHz}$ processor, $4 \mathrm{~GB} \mathrm{RAM}$.

\subsection{Image Data Set}

The coral image data set is very famous image data set for research purpose of image classification and retrieval, in this experimental data set they used 200 images which contain total 10 classes and each class have fixed 20 images from out of coral dataset of thousands images.

\subsection{Result Analysis of Classified Images}

Here in the figure 5.2.1 and figure 5.2.2 compare result for class rose images, where the accuracy measurement of filter based and hybrid-SVM are $85 \%$ and $95 \%$ respectively. Similarly tested on the other nineteen classes, and found the individual and overall accuracy of the proposed hybrid method is higher to the pervious approach.

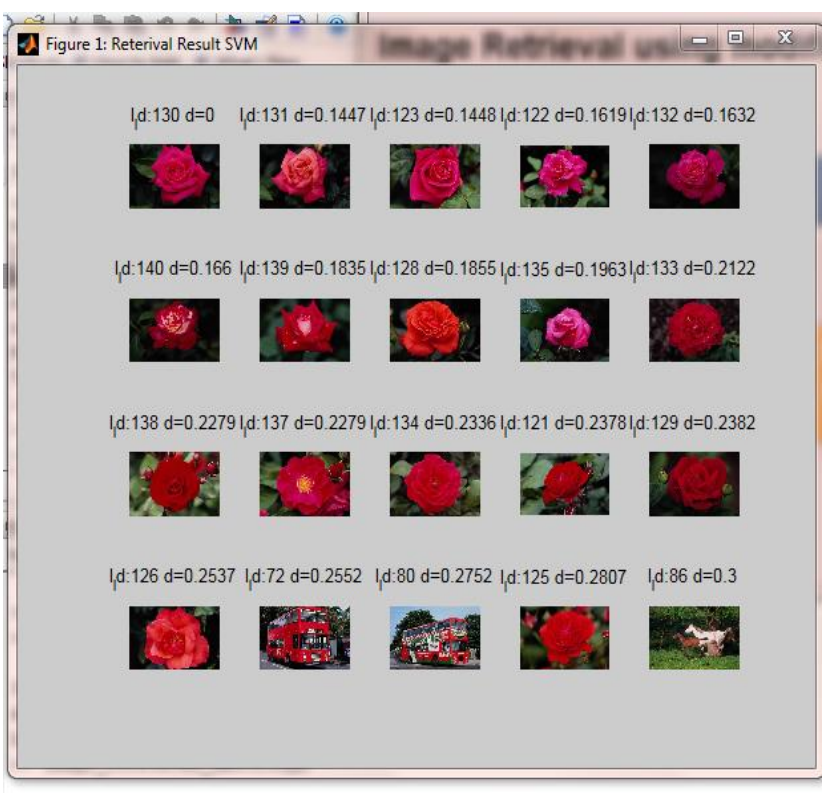

Figure 5.2.1 Filter based methods for rose image 


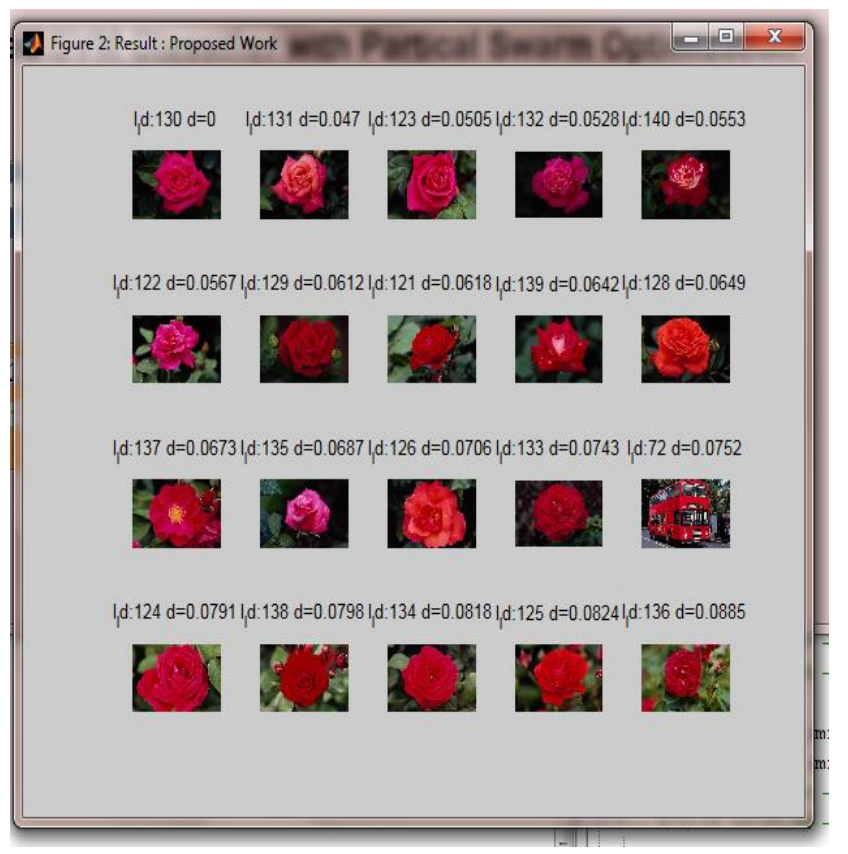

Figure 5.2.2 Hybrid SVM methods for rose image

Similarly they have tested both method in ten different classes, and next section summerized the all classes result.

The result analysis of classified images based on compared two methods with retrieved images. The two tables 5.2.1 and 5.2.2 shows the comparative result analysis of the both methods.

Table 5.2.1 Individual Class wise Accuracy

\begin{tabular}{|c|c|c|}
\hline \multicolumn{3}{|c|}{ Class wise Accuracy } \\
\hline $\begin{array}{c}\text { Image } \\
\text { Class }\end{array}$ & Filterbased & hybridSVM \\
\hline Rural_img & 80 & 95 \\
\hline Beach & 65 & 75 \\
\hline Building & 65 & 80 \\
\hline Buses & 75 & 85 \\
\hline Horse & 95 & 100 \\
\hline Mountains & 50 & 50 \\
\hline Rose & 85 & 95 \\
\hline Elephant & 35 & 40 \\
\hline Dinosaur & 85 & 90 \\
\hline Foods & 80 & 95 \\
\hline
\end{tabular}

Here table 5.2.1 shows that the individual comparative class wise accuracy of both method. After that the figure 5.2.3 shows graph analysis of the individual classes accuracy of the both method, where Hybrid SVM shows the better result up to maximum instant when it compared with Filterbased method.

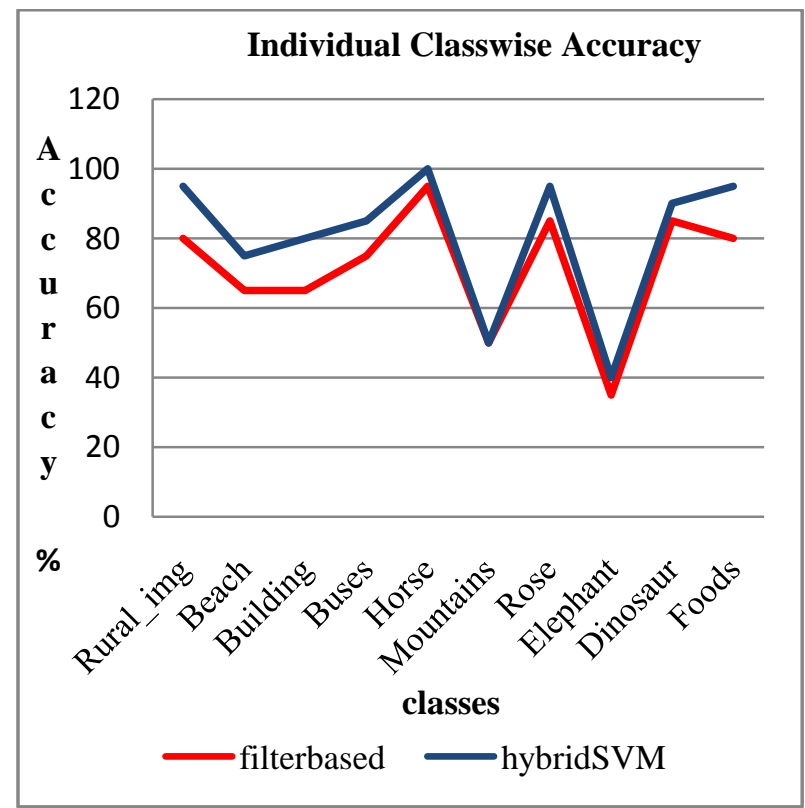

Figure 5.2.3: Individual class accuracies

Here table 5.2.2 shows the overall accuracy of the both methods where the result is examined in the bases of 10 classes of the images dataset. And finally produced the output where Filter based method has overall 71.5 percentage and Hybrid SVM has overall 80.5 percentage accuracies. After that in the figure 5.2.4 its shows that the comparatitive graph analysis of the both method with the help of $3 \mathrm{D}$ bar graph.

Table 5.2.2 Overall Accuracy

\begin{tabular}{|c|c|}
\hline \multicolumn{2}{|c|}{ Overall Accuracy } \\
\hline $\begin{array}{c}\text { Filter Based } \\
\text { method }\end{array}$ & $\begin{array}{c}\text { Hybrid SVM } \\
\text { approach }\end{array}$ \\
\hline 71.5 & 80.5 \\
\hline
\end{tabular}

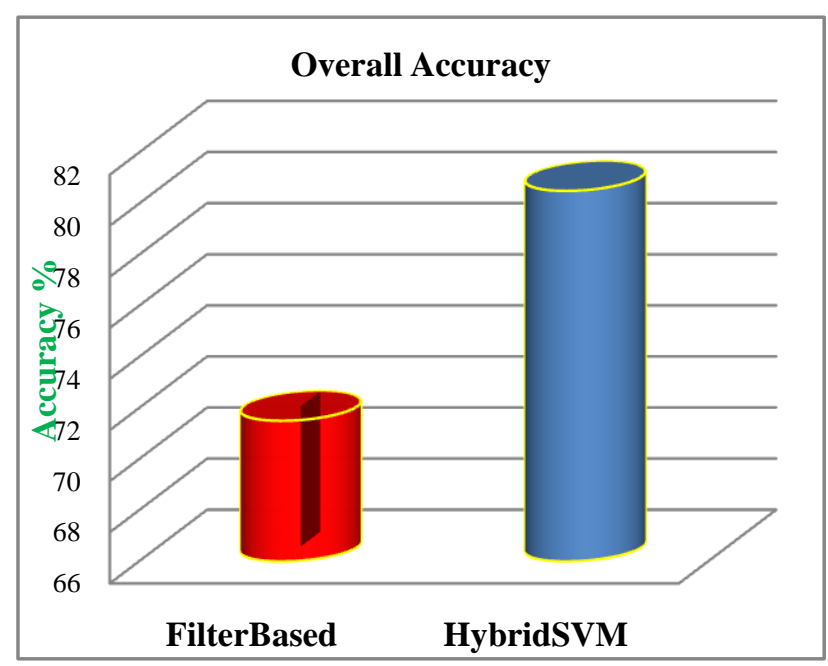

Figure5.2.4 Overall Accuracy 


\section{CONCLUSION AND FUTURE SCOPE}

In this paper novel approach is based on hybrid support vector machine for content-based image retrieval by using modified SVM. It supports more accurate classification rate while processing, and it is compared with filter based method [17]. In the experimental process they used MATLAB2009A and use repudiated coral dataset. After successful experimental setup it was observed that proposed method produces more accurate outcomes and accuracy of proposed method is approx $80.5 \%$ while base method gives $71.5 \%$ accuracy. In future work, the proposed method can be used for the online web search, and can also help for e-commerce product based object searching. Moreover the images used for experimental setup can have noise and some hidden features may not be considered in evaluation of the algorithm. So in future the retrieval result can further be made efficient by removing noise from image dataset.

\section{REFERENCES}

[1] Science, 2012, 5, 32-38 Published Online May 2012 in MECS (http://www.mecs-press.org/) DOI: 10.5815/ijitcs.2012.05.05

[2] K. Ashok Kumar \& Y.V.Bhaskar Reddy, "Content Based Image Retrieval Using SVM Algorithm", international Journal of Electrical and Electronics Engineering (IJEEE) ISSN (PRINT): 2231 - 5284, Vol1, Iss-3, 2012

[3] V. Karpagam, and R. Rangarajan, "Improved contentbased classification and retrieval of images using support vector machine", CURRENT SCIENCE, VOL. 105, NO. 9, 10 NOVEMBER 2013

[4] Chowdhury, M., Das, S. and Kundu, M. K., Novel CBIR system based on ripplet transform using interactive neuro-fuzzy technique. Electron. Lett. Computer Vision Image Anal., 2012, 11, 1-13.

[5] Malik, F. and Baharudin, B. B., Feature analysis of quantized histogram color features for content-based image retrieval based on Laplacian filter. In International Conference on System Engineering and Modeling. IACSIT Press, Singapore, 2012, vol. 34.

[6] K. C. Sia and Irwin King. "Relevance feedback based on parameter estimation of target distribution" In IEEE International Joint Conference on Neural Networks, pages 1974-1979, 2002.

[7] Simon Tong and Edward Chang." Support vector machine active learning for image retrieval. In MULTIMEDIA "in Proceedings of the ninth ACM international conference on Multimedia, pages 107118.2001.

[8] I. Felci Rajam, S. Valli:" A Survey on Content Based Image Retrieval” Life Sci J 2013; 10(2): 2475-2487]. (ISSN: 1097-8135). http://www.lifesciencesite.com 343
[9] Patheja P.S., Waoo Akhilesh A. and Maurya Jay Prakash, "An Enhanced Approach for Content Based Image Retrieval", International Science Congress Association, Research Journal of Recent Sciences, ISSN 2277 - 2502 Vol. 1(ISC-2011), 415-418, 2012.

[10] Sandeep Kumar, Zeeshan Khan, Anuragjain, “A Review of Content Based Image Classification using Machine Learning Approach", International Journal of Advanced Computer Research (ISSN (print): 2249-7277 ISSN (online): 2277-7970) Volume-2 Number-3 Issue-5 September-2012

[11] T. Jyothirmayi, Suresh Reddy, "An Algorithm for Better Decision Tree", (IJCSE) International Journal on Computer Science and Engineering, Vol. 02, No. 09, 2010, 2827-2830.

[12] T. C. Wong, Medical Image Databases. New York, LLC: Springer-Verlag, 1998.

[13] A. Smeulder, M. Worring, S. Santini, A. Gupta, and R. Jain, "Content based image retrieval at the end of the early years", IEEE Trans. Pattern Anal. Machine Intell vol. 22, no. 12, pp. 1349-1380, Dec. 2000.

[14] Efficient Relevance Feedback for Content-Based Image Retrieval by Mining User Navigation Patterns -IEEE transactions on knowledge and data engineering, vol. 23, no. 3, march 2011.

[15] Monika Daga, Kamlesh Lakhwani, "A Novel Content Based Image Retrieval Implemented By NSA Of AIS", International Journal Of Scientific \& Technology Research Volume 2, Issue 7, July 2013 ISSN 2277-8616

[16] Le Hoang Thai, Tran Son Hai, Nguyen Thanh Thuy, "Image Classification using Support Vector Machine and Artificial Neural Network", I.J. Information Technology and Computer.

[17] Mr. S. Manoharan, Dr. S. Sathappan, "A novel approach for content based image retrival using hybrid filter techniques", The 8th International Conference on Computer Science \& Education (ICCSE 2013) April 2628, 2013. Colombo, Sri Lanka.

[18] Swati Agarwal, A. K. Verma, Preetvanti Singh, "Content Based Image Retrieval using Discrete Wavelet Transform and Edge Histogram Descriptor". 2013 International Conference on Information Systems and Computer Networks.

[19] Jisha.K.P, Thusnavis Bella Mary. I, Dr.A.Vasuki “An image retrieval technique based on texture features using semantic properties" . 2013 International Conference on Signal Processing, Image Processing and Pattern Recognition [ICSIPR1. 